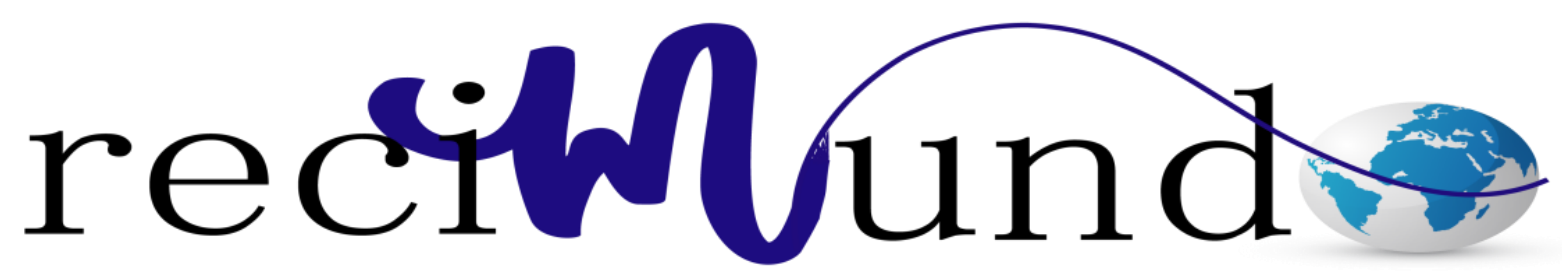

Revista Científica Mundo de la Investigación y el Conocimiento

Alyssa Geslenny Bravo Neira ${ }^{\text {a }}$; Sandra Paola Herrera Macera ${ }^{\text {b}}$; Wendy Judith Álvarez Ordoñez ${ }^{\text {c; }}$ Wladimir Alberto Delgado Conforme ${ }^{\mathrm{d}}$

Traumatismo Craneoencefálico: Importancia de su Prevención y Tratamiento

Cranioencephalic Traumatism: Importance of its Prevention and Treatment

Revista Científica Mundo de la Investigación y el Conocimiento. Vol. 3 núm.2, abril, ISSN: 2588-073X, 2019, pp. 467-483

DOI: $10.26820 /$ recimundo/3.(2).abril.2019.467-483

URL: http://www.recimundo.com/index.php/es/article/view/457

Código UNESCO: 3201 Ciencias Clínicas

Tipo de Investigación: Artículo de Revisión

Editorial Saberes del Conocimiento

Recibido: 15/01/2019

Aceptado: 18/02/2019

Publicado: 31/04/2019

Correspondencia: alygbn@gmail.com
a. Médico; alygbn@gmail.com
b. Médico; sanhema1987@hotmail.com
c. Médico; wenditajao@gmail.com
d. Médico; wladimiroconford@hotmail.com 
Traumatismo Craneoencefálico: Importancia de su Prevención y Tratamiento

Vol. 3, núm. 2., (2019)

Alyssa Geslenny Bravo Neira; Sandra Paola Herrera Macera; Wendy Judith Álvarez Ordoñez; Wladimir Alberto Delgado Conforme

\section{RESUMEN}

El Traumatismo Craneoencefálico (TCE) es una lesión en la cabeza producida por una fuerza externa, la cual es prevenible. Representa un importante problema de salud mundial con altas tasas de mortalidad, según su gravedad, e implica una serie de consecuencias para la calidad de vida del individuo y un gasto considerable para los Estados. La presente investigación plasma la importancia que tiene la prevención y el tratamiento del TCE. Para su desarrollo se llevó a cabo una recopilación y revisión de material documental bibliográfico. Los resultados estuvieron orientados a las causas del TCE y cómo se puede evitar, entre las principales, la formación de los médicos, padres, familiares, representantes o responsables de niños y ancianos, el papel del Estado en la organización y financiamiento de prestación de servicios de salud adecuados, las legislaciones que rigen y el control en materia de violencia y tránsito, y la financiación de la investigación. Asimismo, se recalcó la importancia del diagnóstico precoz y tratamiento adecuado. Es fundamental aunar esfuerzos en todo el mundo para prevenir el TCE, así como mejorar su diagnóstico y tratamiento en pro de una mejor calidad de vida de las personas y un mayor bienestar colectivo a nivel mundial.

Palabras Claves: Importancia; Prevención; Tratamiento; Traumatismo; Craneal. 


\title{
Traumatismo Craneoencefálico: Importancia de su Prevención y Tratamiento
}

Vol. 3, núm. 2., (2019)

Alyssa Geslenny Bravo Neira; Sandra Paola Herrera Macera; Wendy Judith Álvarez Ordoñez;

Wladimir Alberto Delgado Conforme

\begin{abstract}
Cranioencephalic Trauma (TBI) is a head injury produced by an external force, which is preventable. It represents an important global health problem with high mortality rates, according to its severity, and implies a series of consequences for the quality of life of the individual and a considerable expense for the States. The present investigation shows the importance of the prevention and treatment of TBI. For its development a compilation and review of bibliographic documentary material was carried out. The results were oriented to the causes of the TBI and how it can be avoided, among the main ones, the training of doctors, parents, relatives, representatives or responsible for children and the elderly, the role of the State in the organization and financing of service provision of adequate health, the laws that govern and the control in matters of violence and transit, and the financing of the investigation. Likewise, the importance of early diagnosis and adequate treatment was stressed. It is essential to combine efforts throughout the world to prevent TBI, as well as to improve its diagnosis and treatment in favor of a better quality of life for people and greater collective well-being worldwide.
\end{abstract}

Key Words: Importance; Prevention; Treatment; Traumatism; Cranial. 


\section{Traumatismo Craneoencefálico: Importancia de su Prevención y Tratamiento}

Vol. 3, núm. 2., (2019)

Alyssa Geslenny Bravo Neira; Sandra Paola Herrera Macera; Wendy Judith Álvarez Ordoñez; Wladimir Alberto Delgado Conforme

\section{Introducción.}

El traumatismo craneoencefálico (TCE) se define como "cualquier tipo de traumatismo que lleva a una lesión del cuero cabelludo, el cráneo o el cerebro. Las lesiones pueden variar desde un pequeño abultamiento en el cráneo hasta una lesión cerebral grave”. Asimismo, puede clasificarse como cerrado que no es más que cuando la persona recibe un impacto fuerte en la cabeza, causado por un objeto contundente, sin embargo, este no rompe el cráneo. Por otra parte, el TCE abierto que se refiere a la ruptura y/o penetración del cráneo por el impacto, en muchos casos con penetración de objetos extraños. (Biblioteca Nacional de Medicina de los Estados Unidos, 2009)

Los TCE pueden ocurrir como consecuencia de hechos de violencia como un disparo, perforación del cráneo con arma blanca o cuchillo, tubo o bate, entre otros. En el caso de niños pequeños puede ocurrir como consecuencia de maltrato infantil a través de zarandeos o sacudones y en adultos mayores se registra un gran número de TCE consecuencia de caídas. No obstante, el mayor causante de TCE en todo el mundo está constituido por los accidentes de tránsito.

A lo largo de la historia existen innumerables evidencias de la existencia de los traumatismos craneoencefálicos y su tratamiento, desde textos bíblicos y escritos antiquísimos hasta evidencias de estudios en cadáveres antiguos han demostrado cómo el hombre ha sufrido este trauma desde el principio de los tiempos y asombrosamente ha creado la forma de tratarlo para aliviar sus consecuencias. 


\section{Traumatismo Craneoencefálico: Importancia de su Prevención y Tratamiento}

Vol. 3, núm. 2., (2019)

Alyssa Geslenny Bravo Neira; Sandra Paola Herrera Macera; Wendy Judith Álvarez Ordoñez;

Wladimir Alberto Delgado Conforme

En 1912, Julio C. Tello arqueólogo peruano, publicó sus conclusiones acerca del estudio de 200 cráneos trepanados, los cuales se obtuvieron de las tumbas de los Yauyos, comprobando que dichas intervenciones se practicaron como tratamiento de fracturas deprimidas, periostitis, desprendimientos de periostio, lesiones luéticas, fracturas deprimidas y hematomas subdurales, se estima que estas trepanaciones datan de hace 5000 a 2000. Asimismo, una de las pruebas escritas más importantes en la historia del traumatismo craneoencefálico lo constituye el papiro Edwin Smith, el cual fue escrito cerca de 1700 años a.C. y del cual se presume sea una copia de otro escrito entre 3.000 y 2.500 años a.C., en él se describen sistemáticamente cuarenta y ocho casos clínicos, los cuales se inician con temas de trauma de cráneo. (Peña Quiñones, 2010)

Los traumatismos son responsables de más de cinco millones de muertes al año en todo el mundo, una cifra que se podría comparar por las muertes consecuencias del VIH/SIDA, la malaria y la tuberculosis, todas juntas. Asimismo, el estudio fundamental Global burden of disease and risk factores (Carga de morbilidad mundial y factores de riesgo) "calculó que los traumatismos constituían en 1.990 más del $15 \%$ de los problemas de salud en el mundo y preveía que la cifra aumentará hasta el 20\% para 2020”. Por otra parte, se estima que más del $90 \%$ de las muertes por traumatismo se registran en países con bajos y medianos ingresos, los cuales carecen de medidas de prevención, aunado a la escaza preparación de los sistemas de salud para enfrentar este problema. (Gosselin A, Spiegel, Coughlin , \& Zirkle, 2009)

En el caso del traumatismo craneoencefálico en niños, en cuanto a las estadísticas se puede mencionar que, en Estados Unidos de América, para finales de la década de 1.970, estudios demostraron que entre el $13 \%$ y el $30 \%$ de los bebés a los cuales se les diagnostica este traumatismo por maltrato fallecen. Asimismo, indica que un gran número de supervivientes 


\section{Traumatismo Craneoencefálico: Importancia de su Prevención y Tratamiento}

Vol. 3, núm. 2., (2019)

Alyssa Geslenny Bravo Neira; Sandra Paola Herrera Macera; Wendy Judith Álvarez Ordoñez; Wladimir Alberto Delgado Conforme

padecen variados grados de daños que son permanentes, tales como ceguera, discapacidades de aprendizaje y conductuales, parálisis y convulsiones. "El pronóstico de los supervivientes suele ser terrible. Incluso los casos más leves experimentan a menudo dificultades de aprendizaje y tienen problemas para concentrarse. Todos estos pacientes deben someterse a un seguimiento a largo plazo". (Dart \& Cumberland, 2009)

Para Quintana Díaz, García Erce, \& Rodiles Heredia, (2015) citan a Faul M, Xu L, Wald MM, Coronado VG, (2010), cuando refiere que se estima que en "los Estados Unidos de América, de 1,7 millones de personas que sufrirán un TCE en un período de un año, 52.000 de ellos morirán, 27.500 serán hospitalizados y 1.365.000 serán atendidos y dados de alta desde el Servicio de Urgencias”. Asimismo, indican que el grupo de edad a partir de los 65 años, está más propenso a sufrir un TCE y que en aquellas personas de más de 75 años de edad es donde se registran las más altas cifras de Hospitalización, mortalidad y morbilidad. (p. 159)

La importancia del traumatismo craneoencefálico en todo el mundo exige la atención adecuada para disminuir todas sus consecuencias y disfrutar de una mejor calidad de vida. Son los países de ingresos bajos y medianos los que merecen especial atención ya que es donde con menos recursos se cuenta para combatir este grave problema.

El objetivo del presente estudio es plasmar la importancia tanto de la prevención como del tratamiento adecuado y a tiempo del TCE, por cuanto estos dos puntos representan la piedra angular sobre la que se pueden construir los avances en esta materia. 


\section{Traumatismo Craneoencefálico: Importancia de su Prevención y Tratamiento}

Vol. 3, núm. 2., (2019)

Alyssa Geslenny Bravo Neira; Sandra Paola Herrera Macera; Wendy Judith Álvarez Ordoñez;

Wladimir Alberto Delgado Conforme

\section{Materiales y Métodos.}

El presente estudio se realizó mediante una recopilación y revisión de material documental bibliográfico que se utilizó para documentar y plasmar aspectos generales acerca del Traumatismo Craneoencefálico, haciendo énfasis en la importancia de su prevención y tratamiento como bases de una mejor calidad de vida a nivel mundial.

Respecto a la metodología aplicada, Ríos (2017) afirma que la investigación documental “...recurre a la consulta de documentos para obtener sus resultados”. (p. 81).

Por lo tanto, la presente investigación se enmarca en la clasificación de investigación de tipo documental por la fuente usada para su elaboración.

\section{Resultados.}

Prevención y tratamiento

Para Gosselin A, Spiegel, Coughlin , \& Zirkle, (2009) "la comunidad mundial de salud pública todavía no ha asumido, a pesar de haber sido ampliamente documentada, la importancia de prevenir y tratar los traumatismos en los países con ingresos bajos y medios”. Igualmente, exponen que a pesar de que existen datos sumamente fiables acerca de la morbilidad, eficacia, epidemiología y costo-eficacia de muchas otras enfermedades, así como de intervenciones, predomina una carencia de este tipo de información acerca de los traumatismos y su tratamiento.

El impacto y la importancia de los traumatismos no sólo abarca a la salud del ser humano, afectan tanto al paciente o quien la padece como a las personas encargadas de dispensarles el 


\section{Traumatismo Craneoencefálico: Importancia de su Prevención y Tratamiento}

Vol. 3, núm. 2., (2019)

Alyssa Geslenny Bravo Neira; Sandra Paola Herrera Macera; Wendy Judith Álvarez Ordoñez; Wladimir Alberto Delgado Conforme

cuidado necesario, sino que afecta un entorno ambiental intrínseco a infraestructuras y legislaciones en los Estados, abarca los instrumentos causantes como armas (violencia), vehículos (seguridad vial), entre otros.

Formación de los médicos, padres, familiares, representantes o responsables de niños, cuidadores, familiares o responsables de adultos mayores y comunidad en general para la prevención del traumatismo craneoencefálico.

Es fundamental la formación que tengan los médicos y otros trabajadores de la salud en la importancia de la prevención de los traumatismos craneoencefálico, ya que son la base de la construcción de una sociedad consciente de evitar este tipo de accidente y sus consecuencias. Por otro lado, las estadísticas anteriores han descrito como la mayoría de los traumatismos craneoencefálico ocurren en adultos mayores y en gran cantidad de casos en niños pequeños, en donde la responsabilidad de prevenirlos recae sobre sus cuidadores, ya sean, padres, familiares o responsables.

En el caso particular de los traumatismos craneoencefálicos en niños, es importante destacar que se originan en muchos casos por negligencia de sus padre o cuidadores, quienes desconocen la fragilidad del cerebro de estos infantes. La consistencia del cerebro de un bebé pequeño se puede comprar, según su fragilidad, con la gelatina no cuajada, por lo tanto, un zarandeo violento puede hacer mucho más daño que un golpe directo en la cabeza. (Dart \& Cumberland, 2009)

En este sentido, es imperioso crear programas de formación orientados a los padres, representantes o responsables en la atención adecuada a niños de corta edad y evitar el zarandeo 


\section{Traumatismo Craneoencefálico: Importancia de su Prevención y Tratamiento}

Vol. 3, núm. 2., (2019)

Alyssa Geslenny Bravo Neira; Sandra Paola Herrera Macera; Wendy Judith Álvarez Ordoñez;

Wladimir Alberto Delgado Conforme

violento. Asimismo, se requieren políticas que ataquen problemas críticos en todo el mundo como la pobreza, el consumo de drogas, entre otros.

Por medio de estudios realizados en Australia, Canadá y los EE. UU. se ha determinado que el $45 \%$ de los traumatismos craneoencefálico por maltrato eran responsabilidad de los padres biológicos, asimismo, el $25 \%$ era por causa del compañero sentimental de la madre, un $15 \%$ la madre y otro $15 \%$ es responsabilidad de los cuidadores. "En EE. UU., tras la introducción en el estado de Nueva York de un programa hospitalario de educación a progenitores, casos de traumatismo craneoencefálico por maltrato a lactantes se redujeron en $47 \%$ en tres años...”. (Dart \& Cumberland, 2009)

En un estudio realizado por Pinheiro, y otros, (2011) en un Hospital Público de referencia en atención a las víctimas de trauma en la ciudad de Fortaleza-Ceará, a una muestra compuesta por 41 ancianos atendidos con Traumatismo Craneoencefálico durante el periodo de enero a marzo de 2009, en una edad o superior a los 60 años, se concluyó que resulta urgente la prevención de accidentes en la población anciana. Asimismo, se identificó que la principal causa del TCE en estos adultos son las caídas, cuyo lugar predominante de los accidentes fue la calle. (p. 9,10)

En este orden de ideas, es necesario que los cuidadores y familiares, sobre todo de los más ancianos tomen igualmente conciencia en el cuidado y prevención de traumatismos craneoencefálico, adecuando los espacios del hogar a las necesidades del adulto mayor, acondicionándolos en pro de la prevención de cualquier tipo de caídas y no dejándolos solos para brindarles la ayuda y el apoyo en el momento que lo requieran. 


\section{Traumatismo Craneoencefálico: Importancia de su Prevención y Tratamiento}

Vol. 3, núm. 2., (2019)

Alyssa Geslenny Bravo Neira; Sandra Paola Herrera Macera; Wendy Judith Álvarez Ordoñez; Wladimir Alberto Delgado Conforme

La familia, responsables o cuidadores tanto de niños como de adultos mayores pueden ser responsables de disponer para estos un ambiente fuera de riesgos de sufrir TCE. Es de tener en cuenta la seguridad en el automóvil, usar el cinturón de seguridad, revisar las sillas de seguridad de los niños, que tengan buen ajuste, que se encuentren acorde a la edad de estos, el uso de cascos cuando se viaja en bicicleta o moto, son algunos de los factores a tomar en cuenta para prevenir accidentes y traumatismo craneoencefálico.

"Los niños pueden cambiar de asientos de seguridad para automóviles a sillas auxiliares pesando 40 libras o 18 kilogramos. Existen asientos de seguridad para automóviles que están hechos para niños que pesan más de $40 \mathrm{lb}$ o $18 \mathrm{~kg}$ ”. Las leyes relacionadas con las sillas auxiliares para trasladar a niños en automóviles varían de un país a otro. Es ideal para su seguridad trasladar a los niños en sillas auxiliares hasta que tenga al menos 4.9 pies $(145 \mathrm{~cm}) \mathrm{de}$ estatura y alrededor de 8 y 12 años. (Biblioteca Nacional de Medicina de los Estados Unidos, 2018)

El uso del casco se extiende a deportes de contactos, deportes en los que se usan objetos contundentes que puedan golpear la cabeza como el beisbol, al patinar, esquiar o montar a caballo.

Asimismo, debe brindarse a las personas un ambiente seguro en el trabajo, la seguridad laboral es fundamental. En el caso de los niños la seguridad en el hogar no puede faltar, las barandas en escaleras, no dejar solos a los niños más pequeños quienes pueden trepar y caerse, el uso de barandas en la cama y protectores en ventanas pueden brindar un ambiente seguro al niño. 


\section{Traumatismo Craneoencefálico: Importancia de su Prevención y Tratamiento}

Vol. 3, núm. 2., (2019)

Alyssa Geslenny Bravo Neira; Sandra Paola Herrera Macera; Wendy Judith Álvarez Ordoñez;

Wladimir Alberto Delgado Conforme

Igualmente, en el patio de recreo o el colegio deben adoptarse las medidas necesarias para su protección y la prevención de los traumatismos.

El papel de los Estados

La asignación de recursos suficientes para el fortalecimiento de la prestación de servicios médicos (inversión en salud), seguridad vial, seguridad en infraestructura (vías de tránsito, edificios aptos para minusválidos y personas de avanzada edad) son fundamentales para la prevención de los TCE en cada sociedad.

Es de vital importancia que los gobernantes actúen conjuntamente con las comunidades, que sean los directores de los planes de prevención de enfermedades, prevención del riego de enfermarse de sus ciudadanos, deben proporcionar una mejoría en las condiciones de saneamiento e infraestructura, facilitando el acceso a las consultas y la prestación del servicio de exámenes que permitan diagnósticos tempranos que garanticen un rápido tratamiento y por ende, minimicen los riesgos secundarios del TCE.

\section{Legislación}

La legislación es un arma poderosa en la batalla para prevenir el TCE, por medio de una normativa cada país les indica a sus ciudadanos cuáles actos son catalogados como ilícitos o no permitidos y cuál es su sanción o pena, esta es la base para el orden y el bienestar común.

En el caso del TCE por maltrato infantil existen legislaciones que varían de un país a otro pero que concentran el espíritu de la protección al menor. Asimismo, las leyes para la prevención 


\section{Traumatismo Craneoencefálico: Importancia de su Prevención y Tratamiento}

Vol. 3, núm. 2., (2019)

Alyssa Geslenny Bravo Neira; Sandra Paola Herrera Macera; Wendy Judith Álvarez Ordoñez; Wladimir Alberto Delgado Conforme

de traumatismos abarcan las leyes de tránsito, siendo éste uno de los principales causantes del TCE.

La Organización Mundial de la Salud, (2014) publicó un manual de prácticas y recursos para el fortalecimiento de la legislación sobre seguridad vial, donde ofrece a sus países miembros "una hoja de ruta para conseguir una legislación integral relacionada con los cinco principales factores de riesgo de los traumatismos: velocidad, cinturón de seguridad, sistemas de retención infantil, conducción bajo los efectos del alcohol y casco de motociclista...”. (p. 81)

Esta publicación representa un aporte más de la OMS en el fortalecimiento de la prevención del TCE a través del impulso de la creación y control de las diferentes legislaciones en los países.

\section{La financiación de investigaciones}

La inversión o aporte que puedan ofrecer los países, organizaciones y demás interesados a las investigaciones que representen un avance tanto en la prevención como en el diagnóstico temprano del TCE significa una reducción en la tasa de estos accidentes y de sus consecuencias en general.

En tal sentido, el "Comité Especial sobre Investigaciones Sanitarias (OMS) ha promovido desde 1996 un aumento de la financiación para investigaciones traumatológicas, y la segunda edición de Disease control priorities in developing countries ha señalado la investigación y desarrollo sobre traumatología como inversión óptima”. (Gosselin A, Spiegel, Coughlin , \& Zirkle, 2009) 


\section{Traumatismo Craneoencefálico: Importancia de su Prevención y Tratamiento}

Vol. 3, núm. 2., (2019)

Alyssa Geslenny Bravo Neira; Sandra Paola Herrera Macera; Wendy Judith Álvarez Ordoñez;

Wladimir Alberto Delgado Conforme

El Departamento de Salud y Servicios Humanos de EE.UU., (2018) a través de la Administración de Alimentos y Medicamentos de los Estados Unidos (FDA por sus siglas en inglés), indica que son necesarios métodos de diagnóstico más sensibles y objetivos en el trabajo de la detección del TCE leve. Igualmente, indica que un diagnóstico oportuno es fundamental para evitar las lesiones frecuentes y al mismo tiempo coadyuvar en la creación de terapias novedosas. Los científicos de la FDA han diseñado un "modelo de choque para el TCE en animales usando ultrasonido concentrado de alta intensidad y verificaron su precisión, descubrieron que un EEG (medición de actividad eléctrica del cerebro sobre cuero cabelludo) puede detectar el TCE leve en este modelo".

\section{Importancia del tratamiento del TCE}

En un estudio realizado a una población de doscientos sesenta y tres adultos mayores con trauma craneoencefálico, los cuales fueron atendidos por el Servicio de Neurocirugía del Hospital "Manuel Ascunce Domenech" de Camagüey, entre enero del 2002 y enero del 2006, se llegó a la conclusión de que resulta imprescindible para obtener mejores resultados, evitar consecuencias y reducir la tasa de mortalidad en esta población, los protocolos de tratamiento con el propósito de perfeccionar la atención médica. "El tratamiento del adulto mayor con TCE debe ser multidisciplinario, tenaz y activo en el manejo de complicaciones. El diagnóstico precoz y tratamiento oportuno y adecuado pueden incidir satisfactoriamente en el pronóstico de esta enfermedad en este grupo de pacientes". (Mosquera, Vega Basulto, Valdeblánquez, \& Varela Hernández, 2009) 


\section{Traumatismo Craneoencefálico: Importancia de su Prevención y Tratamiento}

Vol. 3, núm. 2., (2019)

Alyssa Geslenny Bravo Neira; Sandra Paola Herrera Macera; Wendy Judith Álvarez Ordoñez; Wladimir Alberto Delgado Conforme

Para Piña Tornés, (2015) si bien es cierto que una política eficaz de prevención es importante, no es menos cierto la importancia que reviste la garantía en el manejo y el tratamiento correcto del TCE, los cuales evitan lesiones cerebrales secundarias, además de identificar anomalías intracraneales las cuales requieran de intervenciones quirúrgicas urgentes. Una correcta atención inicial permite "disminuir la mortalidad precoz, y la calidad de la atención realizada durante los primeros momentos tiene también influencia en el pronóstico final y en las muertes tardías". (p. 153)

La atención prehospitalaria eficaz favorece a la recuperación neurológica del paciente, especialmente en el caso de pacientes graves. "Diversos estudios han destacado la importancia de una correcta valoración inicial de la escala de coma de Glasgow (ECG) en vista de iniciar de inmediato medidas de soporte como el control de la vía aérea”. (Quintana, Rodiles, \& García, 2015)

Por su parte, Cam Páuca (2011) el tratamiento inicial está conformado por los primeros pasos en la terapia para estabilizar al paciente $y$ es fundamental para disminuir las complicaciones secundarias, además de secuelas posteriores, por lo tanto, ha de tomarse en cuenta los siguientes aspectos: “manejo de la vía aérea, estabilización hemodinámica, terapéutica inicial de la hipertensión endocraneana (HIC), sedación y analgesia, uso de anticonvulsivantes y profilaxis de eventos tromboembólicos venosos". Con un adecuado tratamiento inicial se puede prevenir obstrucción de vías aéreas, evitando un aporte deficiente de oxígeno y previniendo la hipercapnia. Asimismo, previene el riesgo de isquemia cerebral secundaria. Previene crisis convulsivas y en el peor de los casos la muerte. (p. 39,43) 


\section{Traumatismo Craneoencefálico: Importancia de su Prevención y Tratamiento}

Vol. 3, núm. 2., (2019)

Alyssa Geslenny Bravo Neira; Sandra Paola Herrera Macera; Wendy Judith Álvarez Ordoñez;

Wladimir Alberto Delgado Conforme

\section{Conclusiones.}

El Traumatismo Craneoencefálico es una de las lesiones más importantes en el mundo motivado a sus altos índices, además de las consecuencias que de ella se derivan incluso a mediano y largo plazo. La secuela va a depender de la intensidad de la lesión y la zona afectada, desde consecuencias leves hasta las más graves, un paciente después de sufrir un TCE puede padecer de cefaleas, mareos, problemas conductuales (depresión, ansiedad...), trastornos del sueño, deterioro cognitivo, pérdida de la memoria, discapacidad (pérdida de la vista, de la movilidad de extremidades, del habla, entre otros) y en el peor de los casos la muerte.

Un traumatismo craneal grave puede tener un lapso de recuperación que va de seis meses a dos años, incluso hasta los 4 años, dependiendo del caso, los costos que esto implica son sumamente elevados, además de las implicaciones en la causa del trauma, violencia, infracción de la ley, accidentes de tránsito, negligencia, impericia, imprudencia, falta de capacitación.

En tal sentido, resulta imperioso resaltar la gran importancia que representa la prevención y el tratamiento del TCE, por cuanto se traduce en múltiples beneficios no sólo a nivel individual sino colectivo. Se requieren estudios actualizados, inversiones en investigación tanto de estadísticas y controles para comparación y ajuste de las medidas actuales a nivel mundial acerca del TCE como investigación en materia de tratamientos efectivos y diagnósticos precoces, a fin de garantizar la prevención de este trauma, la disminución en su incidencia, de sus consecuencias y de la tasa de mortalidad por esta causa. 


\section{Traumatismo Craneoencefálico: Importancia de su Prevención y Tratamiento}

Vol. 3, núm. 2., (2019)

Alyssa Geslenny Bravo Neira; Sandra Paola Herrera Macera; Wendy Judith Álvarez Ordoñez;

Wladimir Alberto Delgado Conforme

\section{Bibliografía.}

Biblioteca Nacional de Medicina de los Estados Unidos. (01 de septiembre de 2009). Medline Plus. Recuperado el 10 de marzo de 2019, de http://www.funsepa.net/medlineplus/spanish/ency/article/000028.htm\#Definici\%C3\%B3 n

Biblioteca Nacional de Medicina de los Estados Unidos. (08 de mayo de 2018). Medline Plus. Recuperado el 17 de marzo de 2019, de https://medlineplus.gov/spanish/ency/patientinstructions/000130.htm

Cam Páuca, J. L. (2011). Manejo inicial del paciente con trauma craneoencefálico e hipertensión endocraneana aguda. Acta Médica Peruana, 28(1), 39-45. Recuperado el 18 de marzo de 2019, de http://www.scielo.org.pe/pdf/amp/v28n1/a07v28n1.pdf

Dart, J., \& Cumberland, S. (mayo de 2009). Cerebro frágil: manéjese con cuidado. Boletín de la Organización Mundial de la Salud, 87, 325-404. Recuperado el 15 de marzo de 2019, de https://www.who.int/bulletin/volumes/87/5/09-030509/es/

Departamento de Salud y Servicios Humanos de EE.UU. (20 de marzo de 2018). Administración de Alimentos y Medicamentos de los Estados Unidos. Recuperado el 17 de marzo de 2019 , de https://www.fda.gov/ForConsumers/ConsumerUpdates/ConsumerUpdatesEnEspanol/uc m575883.htm

Gosselin A, R. A., Spiegel, D., Coughlin , R., \& Zirkle, L. (abril de 2009). Los traumatismos: el problema sanitario desatendido en los países en desarrollo. Boletín de la Organización Mundial de la Salud, 87, 246-246. Recuperado el 12 de marzo de 2019, de https://www.who.int/bulletin/volumes/87/4/08-052290/es/

Mosquera, G., Vega Basulto, S., Valdeblánquez, J., \& Varela Hernández, A. (2009). Protocolo de manejo hospitalario de el trauma craneoencefálico en el adulto mayor. Scielo Cuba. Recuperado el 17 de marzo de 2019, de http://scielo.sld.cu/pdf/amc/v14n1/amc141410.pdf

Organización Mundial de la Salud. (2014). FORTALECIMIENTO DE LA LEGISLACIÓN SOBRE SEGURIDAD VIAL: MANUAL DE PRÁCTICAS Y RECURSOS PARA LOS PAÍSES. Suiza: Biblioteca de la OMS. Recuperado el 17 de marzo de 2019, de https://apps.who.int/iris/bitstream/handle/10665/128039/9789243505107_spa.pdf;jsessio nid=8A2C8D0BFBA173A5FB22A58001555CF0?sequence $=1$

Peña Quiñones, G. (diciembre de 2010). Historia del Trauma Craneoencefálico. (E. OTERORUIZ, Ed.) Revista Academia Nacional de Medic ina, 32(4), 91. Recuperado el 15 de marzo de 2019, de https://encolombia.com/medicina/revistasmedicas/academedicina/vola-91/historiadeltraumacraneoencefalico/ 


\section{Traumatismo Craneoencefálico: Importancia de su Prevención y Tratamiento}

Vol. 3, núm. 2., (2019)

Alyssa Geslenny Bravo Neira; Sandra Paola Herrera Macera; Wendy Judith Álvarez Ordoñez;

Wladimir Alberto Delgado Conforme

Pinheiro, A. I., De Almeida,, F., Barbosa, I., Mesquita Melo, E., Borges Studart, R., \& De Figueiredo Carvalho, Z. (2011). PRINCIPALES CAUSAS ASOCIADAS AL TRAUMATISMO. Enfermería Global, 22, 1-11. Recuperado el 17 de marzo de 2019, de http://scielo.isciii.es/pdf/eg/v10n22/clinica4.pdf

Piña Tornés, A. A. (2015). Manejo del trauma craneoencefálico en la atención primaria en salud. Médicas Vis, 28(1), 153-158. Recuperado el 17 de marzo de 2019, de https://dialnet.unirioja.es/descarga/articulo/5168238.pdf

Quintana Díaz, M., García Erce, J., \& Rodiles Heredia, R. (2015). Traumatismo cráneoencefálico (TCE) y anticoagulación. Archivos de Medicina Interna, 37(3), 158162. Recuperado el 10 de marzo de 2019, de http://www.scielo.edu.uy/pdf/ami/v37n3/v37n3a13.pdf

Quintana, M., Rodiles, R., \& García, J. (2015). Traumatismo cráneo-encefálico (TCE) y anticoagulación. Aspectos esenciales. Archivos de Medicina Interna, 37(3). Recuperado el 17 de marzo de $2019, \quad$ de http://www.scielo.edu.uy/scielo.php?script=sci_arttext\&pid=S1688423X2015000300013

Ríos, R. (09 de 2017). www.eumed.net. (E. Grupo de investigación (SEJ 309) eumed.net de la Universidad de Málaga, Ed.) Obtenido de www.eumed.net: http://www.eumed.net/libros/libro.php?id=1662 